\title{
Chaos Thresholds in finite Fermi systems
}

\author{
P.G.Silvestrov \\ Budker Institute of Nuclear Physics, 630090 Novosibirsk, Russia
}

The development of Quantum Chaos in finite interacting Fermi systems is considered. At sufficiently high excitation energy the direct two-particle interaction may mix into an eigen-state the exponentially large number of simple Slaterdeterminant states. Nevertheless, the transition from Poisson to Wigner-Dyson statistics of energy levels is governed by the effective high order interaction between states very distant in the Fock space. The concrete form of the transition depends on the way one chooses to work out the problem of factorial divergency of the number of Feynman diagrams. In the proposed scheme the change of statistics has a form of narrow phase transition and may happen even below the direct interaction threshold.

PACS numbers: 05.45.+b, 73.23.-b, 71.70.-d

\section{INTRODUCTION}

The investigation of chaotic properties of finite interacting quantum systems has now a few decades history [1] The Random Matrix Theory, originally introduced for modelling this problem, adequately describes the statistics of complicated high energy excitations (see Ref. [8] for an extensive review). However, the concrete mechanism of developing the chaotic behavior in the finite Fermi system with increasing of excitation energy still requires the further understanding. The interest in this problem has even increased in last years in particular due to the recent explosion of activity in the field of Mesoscopic Physics (quantum dots, atomic clusters, helium droplets etc.). The experimental observation of electronic excitations in quantum dots [9] has stimulated the progress 10 14] in theoretical investigation of chaotic disintegration of single electron excitations in this systems. The single particle excitations, being most easily produced experimentally, constitute an important part of Hilbert space of complicated system. However, the number of such states is exponentially small compared to the total number of excited states. The onset of chaos in the majority of many-particle states is the subject of this paper.

We will consider finite, but sufficiently large Fermi systems. The particles are supposed to occupy almost all orbitals below the Fermi energy $\varepsilon_{F}$ of mean field single particle Hamiltonian [15]. The averaged single particle level spacing is $\Delta \ll \varepsilon_{F}$. We will denote as simple states the single Slater determinant states having definite occupation numbers ( 0 or 1 ) of all orbitals. The simple states are mixed by the two-particle interaction and the matrix elements $V$ are supposed to be small and Gaussian random

$$
\overline{V^{2}}=\Delta^{2} / g^{2}, g \gg 1 .
$$

Here $g$ may be interpreted as dimensionless conductance in the case of quantum dot 11, 12]. This simple model was in fact introduced long ago in Refs. [2. 3].

Due to the weak interaction Eq. (11), the low energy excited states are almost unperturbed. With the increase of energy $\mathcal{E}$ the density of states grows rapidly and the exact wave functions at $\mathcal{E} \gg \Delta$ are formed by many chaotically mixed simple states. However, this chaotization of excited states proceeds in a very nonuniform and complicated way. For illustration we have shown on the Fig. 1 different energy scales relevant to this problem.

The complexity of excited state is usually characterized by the inverse participation ratio (IPR) $I=\sum_{k}\left|\psi_{i}(k)\right|^{4}$, where $\psi_{i}(k)$ is the amplitude of $k$-th simple excitation in the $i$-th exact wave function. Physically $I$ is the effective inverse number of simple states mixed into exact one.

While the density of single particle states $\nu_{1}=1 / \Delta$ does not depend on energy, the total density grows extremely fast with $\mathcal{E}$ being $\nu_{\text {total }} \sim \Delta^{-1} \exp (2 \pi \sqrt{\mathcal{E} / 6 \Delta})$ [16]. We use large $\mathcal{E}$ for the total energy of the manyparticle configuration and small $\varepsilon$ for the energy of singleparticle orbitals. Due to the huge number of states (ana$\log$ of the micro-canonical ensemble in thermodynamics) the averaged occupation number for given orbital for arbitrarily chosen "typical" simple state is given by the usual Fermi-Dirac distribution

$$
f\left(\varepsilon-\varepsilon_{F}\right)=\overline{n(\varepsilon)}=\left(\exp \left[\frac{\varepsilon-\varepsilon_{F}}{T}\right]+1\right)^{-1}
$$

where $T=\sqrt{6 \mathcal{E} \Delta} / \pi$ [17]. Note, that we still have not taken into account the interaction $V$, but only explore the wide statistics of single-Slater-determinant states.

The low energy thresholds on the Fig. 1 correspond to the decay of single quasi-particle. Quasi-particles in quantum dot may be seen as peaks broadened by the interaction (bunches of $\delta$-peaks) in the one particle spectral density up to the energy $\varepsilon \sim \Delta g$ 10. Above $\varepsilon \sim \Delta \sqrt{g}$ the broadening arise due to a decay of quasi-particle into two particles and one hole and may be explained by the Fermi golden rule. Below this energy the width predicted by golden rule turns out to be smaller than three-particle level spacing. However, as it was shown recently [12] up to energy $\varepsilon \sim \Delta \sqrt{g / \ln g}$ the quasi-particle disintegration still proceeds through the effective interaction with 
the many excited particle and hole states. A nonuniform distribution of the matrix elements of effective interaction in this case leads to a very unusual distribution of spacings between peaks and widths of the bunches 14.

The original intention of this research was to apply the methods developed for description of quasi-particle disintegration for the investigation of the full spectrum of complicated system. However, the onset of chaos in the many-particle excitations provided us with a number of surprising new effects not present in the single quasiparticle case. Only the estimate of the effective high order matrix element in the section 3 may be considered as straightforward generalization of the analogous estimate in the Ref. 14]. In the section 2 we will show, how the direct interaction between particles may mix into one wave function an exponentially large number of simple states even inside the region of Poissonian spectrum. In the section 3 we consider the self-consistent scheme of onset of chaos based on the analysis of the scaling behavior of the direct and effective interaction. However, in the following section 4 we propose an another scenario of the transition to Wigner-Dyson caused by the factorial divergency of the high order interaction. The detailed comparison of the features of these two scenarios is given at the end of paper (Sec. 5).

\section{MIXING BY THE DIRECT INTERACTION}

For weak interaction the strong mixing of different quantum states may take place only if their energies occasionally turn out to be very close. Therefore, consider first only two states $|i\rangle$ and $|f\rangle$ with energy difference $\varepsilon$ connected by matrix element $V$. The simple calculation [18] gives the IPR for exact wave function $\alpha|i\rangle+\beta|f\rangle$

$$
I=\alpha^{4}+\beta^{4}=1-\frac{2 V^{2}}{\varepsilon^{2}+4 V^{2}} .
$$

Let us fix the state $|i\rangle$ and consider a set of states $|f\rangle$ with a density $d n / d \varepsilon=\Delta_{f}^{-1}$. It is convenient to introduce the intermediate scale $\varepsilon_{0} ;|V| \ll \varepsilon_{0} \ll \Delta_{f}$. With the probability $1-2 \varepsilon_{0} / \Delta_{f}$ there are no final states falling into the strip $-\varepsilon_{0}<\varepsilon<\varepsilon_{0}$ and $I=1$ up to negligible correction $\sim V^{2} / \Delta_{f} \varepsilon_{0}$. With the small probability $2 \varepsilon_{0} / \Delta_{f}$ one of the states $|f\rangle$ enters the strip. In order to find $I$ in this case one has to average the Eq. (3) over the interval of energy

$$
\int_{-\varepsilon_{0}}^{\varepsilon_{0}}\left(1-\frac{2 V^{2}}{\varepsilon^{2}+4 V^{2}}\right) \frac{d \varepsilon}{2 \varepsilon_{0}}=1-\frac{2 \pi|V|}{2 \varepsilon_{0}}
$$

and then average the $|V|$ over the ensemble of matrix elements. Combining the two contributions one finds

$$
I=1-\frac{2 \varepsilon_{0}}{\Delta_{f}}+\frac{2 \varepsilon_{0}}{\Delta_{f}}\left(1-\frac{2 \pi \overline{|V|}}{2 \varepsilon_{0}}\right)=1-2 \pi \frac{\overline{|V|}}{\Delta_{f}} .
$$

This simple formula is basic for our perturbative estimates. The averaging of the modulus of $V$ for strongly non-Gaussian high order effective matrix elements will be the main subject of the next section.

The goal of this paper is to consider the mixing of complicated excited states. Occupation numbers for these states are described by the Eq. (2). The density of directly accessible states, or the density of pairs of occupied 1,2 and unoccupied 3,4 states with $\varepsilon_{1}+\varepsilon_{2}=\varepsilon_{3}+\varepsilon_{4}$ which may be connected directly by the interaction is

$$
\frac{1}{\Delta_{2}}=\frac{1}{(2 !)^{2}} \int \delta\left(\sum x_{i}\right) \prod_{i=1}^{4} f_{i} \frac{d x_{i}}{\Delta}=\frac{T^{3}}{\Delta^{4}} \frac{\pi^{2}}{6} .
$$

Here $f_{i}=f\left(x_{i}\right)$ (2), $x_{1,2}=\varepsilon_{1,2}-\varepsilon_{F}, x_{3,4}=\varepsilon_{F}-\varepsilon_{3,4}$ and we have used that $1-f(x)=f(-x)$. The factor $(2 !)^{-2}$ accounts for the identical initial and final particles. It is easy now to use the Eq. (5) in order to find the IPR. However, this result may be even further improved. Eq. (5) with $\Delta_{f}=\Delta_{2}$ describes the situation when only for one two-by-two transition $1,2 \rightarrow 3,4$ the energy difference turns out to be of the same order of magnitude with the matrix element. In the second order in $V$ the leading contribution to $I$ is given by double event $1,2 \rightarrow 3,4$ and $5,6 \rightarrow 7,8$. However, as far as none of the orbitals $5-8$ not coincides with any of $1-4$ these two corrections are completely independent and corresponding contributions to $I$ should be simply multiplied. Effectively, all one-particle states are chosen from $\sim T / \Delta$ orbitals around $\varepsilon_{F}$. Therefore, if the number of couples of pairs falling into the energy interval $\sim|V|$ is small compared to $\sim T / \Delta$ one may neglect the interference between different direct transitions. Again we may introduce the intermediate scale $\varepsilon_{0} ;|V| \ll \varepsilon_{0}$. The number of directly accessible final states is $2 \varepsilon_{0} / \Delta_{2} \gg 1$, but until $\varepsilon_{0} / \Delta_{2} \ll T / \Delta$ the IPR is found simply as a product over these channels

$$
\begin{aligned}
I=\left(1-2 \pi \frac{\overline{|V|}}{2 \varepsilon_{0}}\right)^{\frac{2 \varepsilon_{0}}{\Delta_{2}}} & =\exp \left\{-2 \pi \frac{\overline{|V|}}{\Delta_{2}}\right\} \\
& =\exp \left\{-\frac{4 \sqrt{3}}{\sqrt{\pi} g}\left(\frac{\mathcal{E}}{\Delta}\right)^{\frac{3}{2}}\right\} .
\end{aligned}
$$

Here we have averaged $|V|$ for the Gaussian random interaction Eq. (11). This result is valid for $\Delta / g \ll \Delta_{2} T / \Delta$, or $T \ll \Delta \sqrt{g}$. The exponentiation of the IPR is a direct consequence of the two-particle character of the interaction. It would be very inconvenient to investigate such effects if one will consider the Hamiltonian of the system as a random (sparsed) Matrix in the full Hilbert space. Surprisingly, to the best of my knowledge, the simple effect Eq. (7) has not been considered yet e.g. for description of the excitations in complex nuclei.

Due to the Eq. (7) at $\mathcal{E}>\Delta g^{2 / 3}$ the number of components in typical wave function becomes exponentially 
large. However, within the range of validity of the Eq. (7) only a small (also exponentially) part of close energy levels is mixed in any exact eigenstate. Therefore this simple effect is not enough for creation of full Quantum Chaos.

\section{HIGH ORDER EFFECTIVE INTERACTION}

Corrections to the Eq. (7) arise after taking into account of the high order effective interaction between three-particle states, then four-particle etc. The example of corresponding tree-type Feynman diagram is shown on Fig. 2. The tree-type diagrams are maximally enhanced due to a wide statistics of initial and final orbitals. Contributions from the diagrams with closed loops are small like some power of $\Delta / T$. Generalization of the Eq. (6) for the case of $n$-particle interaction gives

$$
\frac{1}{\Delta_{n}}=\int \frac{\delta\left(\sum x_{i}\right)}{(n !)^{2}} \prod_{i \leq 2 n} f_{i} \frac{d x_{i}}{\Delta}=\frac{(2 \pi T)^{2 n-1}}{\pi n(2 n) ! \Delta^{2 n}}
$$

Again $(n !)^{-2}$ accounts for the identical fermions. Note that $(2 n) ! \sim 2^{2 n}(n !)^{2}$ and therefore the integration over the energies $x_{i}$ does not lead here to any $n$ ! suppression. This is in strict contrast with what happens for decay of quasi-particle 14], where the main suppression of the density of final states $\nu_{n}$ arose due to the integration over the energies of final particles.

Sufficiently more complicated turns out to be the calculation of averaged modulus of the effective matrix element entering both the Eq. (5) and the Eq. (7). The simplest variant of the effective interaction is given by the three-particle interaction arising in the second order of perturbation theory

$$
V_{e f f}^{(3)}=\sum_{2} \frac{V_{12} V_{23}}{\varepsilon_{1}-\varepsilon_{2}} .
$$

Formally this interaction mixes together three states $|1\rangle$, $|2\rangle,|3\rangle$. The use of the concept of effective interaction requires that the admixture of the intermediate state $|2\rangle$ in the total wave function is small compared to those of $|1\rangle$ and $|3\rangle$. This means that $V_{12}, V_{23} \ll\left|\varepsilon_{1}-\varepsilon_{2}\right| \approx\left|\varepsilon_{2}-\varepsilon_{3}\right|$. The contribution to averaged IPR from strongly mixed (almost degenerate) $|1\rangle,|2\rangle$ and $|3\rangle$ should be considered separately, but this correction is small like $1 / \ln g$ compared to the total contribution of the effective interaction. On the other hand, we will be interested in the tail of distribution of the effective matrix elements. For Gaussian distributed $V$ (1) the probability to find a large individual matrix element of the original two-particle interaction is exponentially small. The large matrix elements appears if one term in the sum in Eq. (9) occasionally has anomalously small denominator $\left|\varepsilon_{1}-\varepsilon_{2}\right| \ll \Delta$. All the other (random) contributions in the sum in this case may be neglected for $\ln g \gg 1$. The result of this lengthy discussion may be summarized in the formula for the probability distribution for the effective matrix element (9)

$$
P^{(3)}(V) \sim \frac{\Delta}{g^{2}} \frac{1}{V^{2}} ; g^{-2}<|V| / \Delta<g^{-1} .
$$

The averaged modulus of the matrix element in this case is $\overline{\left|V_{e f f}^{(3)}\right|} \sim \Delta \ln g / g^{2}$.

Higher order effective matrix elements are obtained by simple adding of more intermediate states into the Eq. (9). The extensive discussions of the problems arising while calculating the averaged value of this matrix element may be found in Ref. 114. For example, the averaged modulus of the four-particle effective matrix element for given Feynman diagram is given by

$$
\begin{aligned}
\overline{\left|V_{e f f}^{(4)}\right|}= & \overline{\sum_{2,3} \frac{V_{12} V_{23} V_{34}}{\varepsilon_{2} \varepsilon_{3}} \mid}= \\
& \frac{(\sqrt{2} \Delta)^{3}}{(\sqrt{\pi} g)^{3}} \int \frac{1}{\left|\varepsilon_{2} \varepsilon_{3}\right|} \frac{d \varepsilon_{2}}{2 \Delta} \frac{d \varepsilon_{3}}{2 \Delta} .
\end{aligned}
$$

Here compared to the Eq. (9) we have made the change of variables $\varepsilon_{1}-\varepsilon_{2} \rightarrow \varepsilon_{2}$ and $\varepsilon_{1}-\varepsilon_{3} \rightarrow \varepsilon_{3}$. The preintegral factor $\sim(\Delta / g)^{3}$ in the Eq. (11) arose after averaging of Gaussian distributed (1) direct matrix elements $\left|V_{i j}\right|$. The upper bound for the both remaining integrals in the Eq. (11) is given by the single-particle level spacing $\left|\varepsilon_{2,3}\right|<\Delta$. Due to the logarithmic character of the integrals the order of magnitude estimate of the bounds is enough. More interesting is the origin of the bounds at small $\varepsilon_{2,3}$. Analogously to what we have discussed for the three-particle interaction the formula (11) describes the mixing of four states $|1\rangle,|2\rangle,|3\rangle,|4\rangle$ in the third order of perturbation theory. The interaction of the four states may be replaced by the effective interaction of only two $|1\rangle$ and $|4\rangle$ if the admixture of intermediate states $|2\rangle$ and $|3\rangle$ is small. This requirement for example for state $|2\rangle$ means that $\left|\varepsilon_{2}\right| \gg\left|V_{12}\right| \sim \Delta / g$ (weak mixing of $|2\rangle$ and $|1\rangle$ ) and $\left|\varepsilon_{2}\right| \gg\left|V_{23} V_{34} / \varepsilon_{3}\right| \sim(\Delta / g)^{2} /\left|\varepsilon_{3}\right|$ (weak mixing of $|2\rangle$ and $|4\rangle)$. Another two inequalities for the vector $|3\rangle$ are $\left|\varepsilon_{3}\right| \gg \Delta / g$ and $\left|\varepsilon_{3}\right| \gg(\Delta / g)^{2} /\left|\varepsilon_{2}\right|$. Combining all this inequalities one finds for the range of integration in the Eq. (11)

$$
\Delta / g \ll\left|\varepsilon_{2,3}\right| \ll \Delta .
$$

These bounds allows one to find the averaged matrix element with the $\sim 1 / \ln g$ accuracy: $\overline{\left|V_{e f f}^{(4)}\right|} \sim \Delta(\ln g)^{2} / g^{3}$. In the similar way one may consider the five-particle, six-particle, etc. effective interaction. Unfortunately, the system of inequalities describing the range of integratin for the intermediate energies turns out to be sufficiently more complicated in these cases.

The formulas like Eq. (11) may be used to find again the distribution of the effective matrix elements. In particular for the four-particle interaction 


$$
\begin{aligned}
& \overline{\left|V_{\text {eff }}^{(4)}\right|}=\int P^{(4)}\left(V_{\text {eff }}^{(4)}\right) d V_{\text {eff }}^{(4)}, \\
& \quad P^{(4)}\left(V_{\text {eff }}^{(4)}\right)= \\
& \frac{(\sqrt{2} \Delta)^{3}}{(\sqrt{\pi} g)^{3}} \int \delta\left(V_{\text {eff }}^{(4)}-\frac{(\sqrt{2} \Delta)^{3}}{(\sqrt{\pi} g)^{3}} \frac{1}{\varepsilon_{2} \varepsilon_{3}}\right) \frac{d \varepsilon_{2} d \varepsilon_{3}}{4\left|\varepsilon_{2} \varepsilon_{3}\right| \Delta^{2}} .
\end{aligned}
$$

Here the range of variation of $\varepsilon_{2}$ and $\varepsilon_{3}$ is the same as in the Eq. (11) Found in this way the probability distribution in the general case has the form

$$
P^{(n)}(V) \sim \frac{\Delta}{g^{n-1}} \frac{1}{V^{2}} \chi^{(n)} ; g^{-n+1}<|V| / \Delta<g^{-1},
$$

where $\chi^{(n)}(V)$ is a piecewise polynomial function of the order $n-3$ of two logarithms $\ln (|V| / \Delta)$ and $\ln g$. Again in the simplest non-trivial example of $n=4$ one finds

$$
\chi^{(4)}=\left\{\begin{array}{l}
\ln \left(|V| g^{3} / \Delta\right), g^{-3}<|V| / \Delta<g^{-2} \\
-\ln (|V| g / \Delta), g^{-2}<|V| / \Delta<g^{-1}
\end{array} .\right.
$$

Also notice the huge range of variation of $V_{e f f}^{(n)}$ (14) for large $n$. Averaging of the matrix element over the distribution Eq. (14) leads to

$$
\overline{\left|V_{e f f}^{(n)}\right|} \sim \Delta(\ln g)^{n-1} / g^{n} .
$$

Due to a complicated domain of integration over the intermediate energies it is difficult even to estimate the value of the overall numerical constant here. However, the important result of Ref. [14] is that at large $n$ this unknown overall constant is neither large like $n$ ! nor small like $1 / n$ !. The appearance of logarithms in the coefficients of wave function was also observed in 19 .

Like in Eq. (7) the corrections generated by the many particle effective interaction are naturally exponentiated at least for $n \ll T / \Delta$. Thus combining $\Delta_{n}(\mathbb{8})$ with $V_{\text {eff }}^{(n)}$ (16) we obtain instead of the Eq. (7)

$$
\begin{gathered}
I=\exp \left\{-\frac{4 \sqrt{3}}{\sqrt{\pi} g}\left(\frac{\mathcal{E}}{\Delta}\right)^{3 / 2} F\left(\frac{\ln g}{g} \frac{\mathcal{E}}{\Delta}\right)\right\}, \\
F(x)=1+\sum F_{n} x^{n} .
\end{gathered}
$$

In the case of short range screened two-particle interaction $V\left(\vec{r}_{1}, \vec{r}_{2}\right) \sim \delta\left(\vec{r}_{1}-\vec{r}_{2}\right)$ the first coefficient here is $F_{1}=24 \sqrt{2 / \pi} / 5$. This result for $I$ may be compared with the perturbative result for the IPR for disintegration of the single quasi-particle in the isolated Quantum dot found in Refs. 12 14

$$
I_{q}=1-\frac{1}{\ln g} \phi(y) \quad, \quad \phi=\sum_{n=1}^{\infty} \phi_{n} y^{n},
$$

where $y=(\varepsilon / \Delta)^{2} \ln g / g$ and $\varepsilon$ is the energy of quasiparticle. By analogy with the usual IPR, the $I_{q}$ is a sum of fourth powers of the amplitudes to find given $k$ th quasi-particle in the $i$-th exact state $I_{q}=\sum_{i}\left|\psi_{i}(k)\right|^{4}$. The next section will be devoted to the more detailed investigation of the possible properties of the scaling function $F(x)$. For single quasi-particle the coefficients $\phi_{n}$ decrease like $n !^{-1}$ for large $n$, which makes very improbable the disintegration of quasi-particle through delocalization transition in the Fock space.

The simple counting of the powers of $\mathcal{E} / \Delta$ and $g$ in Eq. (17) leads to the following scenario of the development of chaos:

a). For $\Delta g^{2 / 3}<\mathcal{E}<\Delta g / \ln g$ the Eq. (7) is valid. The number of components in typical wave function is exponentially large, but described by simple multiplication of independent direct transitions.

b). At $\mathcal{E} \sim \Delta g / \ln g$ all terms in the series Eq. (17) enters the game. The wave function is formed by a fractal combination of states distant in the Fock space [20]. If the resumed scaling function $F$ will be a regular function of the argument $x$ (like it is for the $\phi(y)(18)$ ), the number of components of the wave function will be small compared to the total density of states and the level statistics will be close to Poissonian.

c). Starting from $\mathcal{E} \sim \Delta g$ all quasi-particles constituting the wave function have enough energy to decay via usual Golden-rule. Only here the Wigner-Dyson statistics is evident. The more formal criteria for onset of chaos will be the requirement that the product $I \mathcal{E} \nu_{\text {total }}$ became of the order of $\sim 1$, where $\nu_{\text {total }}$ is the total density of states described above the Eq. (2). This condition is equivalent to the equation for unknown function $F(x)$

$$
x F(x)=\frac{\pi \sqrt{\pi}}{6 \sqrt{2}} \ln g .
$$

Solution of this equation gives the energy of transition to rigid spectrum somewhere within $g / \ln g<\mathcal{E} / \Delta<g$. Due to the exponential character of $I$ and $\nu_{\text {total }}$ most likely this will be the narrow phase transition.

\section{THE $N$ ! SCENARIO}

Thus the power counting for the perturbative IPR Eq. (17) may provide us with the formally self-consistent scenario of developing of chaos in finite Fermi systems. Now we are going to show, how taking into account of the high order behavior of the coefficients $F_{n}$ may lead to the important revision of this straightforward scheme. Unfortunately, we are not able to perform the complete estimation of the asymptotics of $F_{n}$. Nevertheless, the catastrophic change of the mechanism of onset of chaos, which we will consider, may partly compensate the lack of rigor. Therefore, even though based on certain assumptions, the result of this section should be considered as one of the main results of the paper. 
The calculation of any term of the series in Eq. (17) consists of two main steps. First is the calculation of the density of final states, which we were able to perform explicitly (8). The second step is the estimation of the effective matrix element. The matrix element for a given Feynman diagram is described by the Eq. (16). As we have told, this matrix element could not contain any $n$ ! or $n !^{-1}$. However, for many-body problem and high order of perturbation theory the same initial and final configurations may be connected by many different Feynman diagrams. We have shown on the Fig. 2 the example of corresponding tree-type $n$-particle diagram. The total number of diagrams like on Fig. 2 is

$$
\text { \#diagrams }=N_{d}=n^{n-2}(n-1) ! n ! .
$$

Here $n$ ! is the number of permutations of $1^{\prime}-n^{\prime}$ final particles, $(n-1)$ ! is the number of replacements of (screened) Coulomb interaction lines and $n^{n-2}$ is the number of $(n-1)$-segment trees connecting $n$ points $1-n$ 21]. Eq. (20) gives us the number of diagrams of Schrödinger perturbation theory. Among the $N_{d}$ trees there are many diagrams having the same set (up to permutations) of the matrix elements of two-particle interaction $V_{i j}$ and different energy denominators. As it was pointed out in Ref. [14], these diagrams could not be considered as statistically independent, which results in lowering of the mixing by the high order effective interaction. The averaged modulus of the effective matrix element found for single Feynman diagram (16) should be modified in the case of many interfering diagrams. However, due to a huge range of variation of the $V_{\text {eff }}$ in the power law distribution Eq. (14) the average value is saturated by a rare very large fluctuations of the matrix element. Therefore for $n$ not too large the generalization of the Eq. (16) for many diagram case is achieved by the simple multiplication by the number of diagrams (we will return later to the discussion of very large $n$ ). Finally, combining the Eqs. (8), (16) and (20) we find

$$
F_{n} \leq n ! .
$$

We see, that the series in Eq. (17) most likely is the asymptotic series. Due to the unsatisfactory estimate of the number of diagrams and even the value of individual diagram we were able to draw only the upper bound for the $F_{n}$. However, it seems very unlikely that the cancellation between the diagrams of the Schrödinger perturbation theory will remove the factorial divergency of the coefficients of the series. In the following (if the opposite not indicated explicitly) we suppose that $F_{n} \sim n$ !. The asymptotic series, common in the Field theory and High Energy physics, usually do not cause serious troubles (see for review Ref. [22]). In all known cases one simply breaks the summation on the smallest term of the series and use this smallest term also as the order of magnitude estimate of the rest nonperturbative part of the sum. The more refined strategy will be to perform the Borel summation, but this procedure (up to the same nonperturbative corrections) is equivalent to the breaking of the series at the smallest term. In our example this means that one should break the summation in the Eq. (17) at $n_{0} \sim g \Delta / \ln g \mathcal{E}$ and the non-perturbative ambiguity in $F$ should be

$$
\delta F_{n p} \sim e^{-n_{0}} \sim \exp \left\{-\sigma \frac{g}{\ln g} \frac{\Delta}{\mathcal{E}}\right\},
$$

with some $\sigma \sim 1$. However, in the known field-theoretical examples there exist the clear physical mechanisms (Instanton, Renormalon) which allows to understand the origin of non-perturbative corrections and the necessity of breaking the series at the smallest term. In our manybody quantum problem we could find no physical motivation for the appearance of nonperturbative corrections of the kind (29) and we see no reasons for breaking the summation in the Eq. (17) at the smallest term. Of course, the Eq. (17) is not exact, but corrections to $F(x)$ are small like $(\ln g)^{-1}$ or $\Delta / \mathcal{E}$. Taking into account such corrections will help us, to sum up the original divergent series. Nevertheless, in order to make sense of our perturbative analysis of the onset of chaos in finite Fermi systems we should find the physically motivated cutoff for the $n$ ! divergent sum. The natural cutoff for $F_{n}$, which we see, is given by the total number of particles which may enter into the diagram of effective interaction $n_{\max } \sim T / \Delta \sim \sqrt{\mathcal{E} / \Delta}$. If $n_{\max }>x^{-1}$ the summation now goes above the smallest term of the series. The consequences of the decision to sum up the series above the smallest term are crucial. In the standard scheme the function $F(x)$ is described by perturbation theory at small $x$ and became completely nonperturbative (although it still may be smooth and finite) at $x \sim 1$. Within the new scenario $F(x)$ blows up at

$$
F_{n_{\max }} x^{n_{\max }} \sim\left(n_{\max } \ln g \mathcal{E} / g \Delta\right)^{n_{\max }} \sim 1,
$$

which

gives the chaotization threshold $\mathcal{E}_{c} \sim \Delta(g / \ln g)^{2 / 3}$. This estimate was done for the most strongly divergent series allowed by the Eq. (21). For example, if $F_{n} \sim(n / 2)$ ! the same calculation will lead to $\mathcal{E}_{c} \sim \Delta(g / \ln g)^{4 / 5}$. Therefore, the more accurate conclusion from the Eq. (21) is that due to the effective interaction including all $\sim T / \Delta$ excited particles the series for IPR Eq. (7) blows up at some $(g / \ln g)^{2 / 3}<\mathcal{E}_{c}<g / \ln g$ [23].

The two important peculiarities of the emergence of Quantum Chaos follow from this "factorial" scenario:

I. The direct interaction connect only the states close in the Fock space [20]. Therefore, the Eq. (7) describes mixing of only a small part of close levels. On the other hand, the highest order effective interaction entering the Eq. (23) mixes the huge number (the majority of all many-particle excitations) of very distant simple states. 
Thus, divergency of $F(x)$ due to the $n$ ! leads to the transition from Poisson to rigid (Wigner-Dyson) spectrum at $\mathcal{E}=\mathcal{E}_{c}$.

II. Due to the large power of $x$ in the Eq. (23) the growth of $F(x)$ with increase of energy takes place at a very narrow interval $\Delta \mathcal{E} / \mathcal{E}_{c} \sim 1 / n_{\max }$. This means that the change of statistics has a form of phase transition with relative width $\sim 1 / n_{\max }$.

Our description of the development of chaos is sufficiently based on the estimate of $n_{\max }(23)$. If one would be able to find another reasons for breaking the series at a term parametrically smaller than $n_{\max } \sim T / \Delta$ the result for $\mathcal{E}_{c}$ will be also different. For example, the new cutoff may arose due to a huge number of interfering diagrams Eq. (20). The joint probability distribution for the sum of many diagrams with power law individual distributions described by the Eq. (14) has a Lorentzian form [24] with the width $\gamma$ having the form

$$
P(V) \sim \frac{\gamma}{V^{2}+\gamma^{2}} \frac{\chi(V)}{\chi(\gamma)} ; \gamma \sim \chi(\gamma) \frac{\Delta N_{d}}{g^{n-1}} \sim \Delta \frac{n^{3 n}}{g^{n}} .
$$

Here we have used for the estimate of the number of independent diagrams the upper bound Eq. (20). This distribution is valid for $|V|<\Delta / g$. The function $\chi$ was defined in the Eq. (14). The factor $\chi(V) / \chi(\gamma)$ allows to preserve the asymptotics Eq. (14) at $|V| \gg \gamma$. Due to the logarithmic character of $\chi$ this factor correctly accounts for the deviation from the pure power law in the distribution of single matrix elements. We see that after taking into account of the interference of different diagrams the power law distribution of the total matrix element becomes strongly violated at $|V| \sim \gamma$, not at $|V| \sim \Delta g^{-n+1}$ as in the Eq. (14). The value of $F_{n}$ found with the distribution $P(V)$ (24) becomes parametrically different from the estimate Eq. (21) only at $n_{\text {max }}^{\prime} \sim g^{1 / 3}$ (even at $n_{\text {max }}^{\prime} \sim(g / \ln g)^{1 / 3}$ if one considers seriously the high powers of logarithms in the Eq. (24)). However, this new variant of the cutoff leads to the same threshold for the onset of chaos as we have found before in Eq. 23. This coincidence of the results of two different approaches to estimation of the $n_{\max }$ may be considered as an indirect support for the transition to Wigner-Dyson statistics at $\mathcal{E}_{c} \sim \Delta(g / \ln g)^{2 / 3}$.

At least some of the thresholds shown on the Fig. 1 may lie above $\mathcal{E}_{c}$. The physical meaning of these perturbative thresholds becomes less clear. Nevertheless, even above $\mathcal{E}_{c}$ one may try to look for effects generated by the direct or low order effective interaction. The highest order effective interaction becomes important due to the huge combinatorics Eq. (20), but the corresponding matrix element is in general very small (up to $V \sim \Delta / g^{n_{\max }}$ ). Thus this high order interaction may be invisible (or at least suppressed) at small time in time-dependent problems. For example, one may consider the time evolution of the wave packet $\Psi(t)$, starting at $t=0$ from single
Slater determinant. The pure direct interaction in this case gives

$$
|\langle\Psi(0) \mid \Psi(t)\rangle|^{2}=e^{-\Gamma t} ; \Gamma=2 \sqrt{6} \Delta g^{-2}(\mathcal{E} / \Delta)^{3 / 2} .
$$

The formal power counting (like we have done below the Eq. (17)) gives for the range of validity of this result $\Delta g^{2 / 3} \ll \mathcal{E} \ll \Delta g / \ln g$. However, due to the logarithmic behavior of all integrals (see e.g. the Eq. (11) all values of the high order effective matrix elements are equally important within $g^{-n+1}<|V| / \Delta<g^{-1}$ (14). Therefore, the range of validity of the Eq. (25) may be changed strongly after taking into account of the contribution from large high order effective matrix elements.

\section{DISCUSSION}

The goal of this paper was to consider, how the chaotic mixing of noninteracting eigenstates develops with increasing the excitation energy in finite weakly interacting Fermi systems. Measured in the units of the singleparticle level spacing $\Delta$ the energy corresponding to the onset of rigid spectrum may depend on only one parameter $g$ (11). Nevertheless, even with only one parameter we were able to introduce a number of different energy scales, shown on the Fig. 1, which correspond to (or may correspond to) the different stages of development of chaos.

The low lying thresholds on the figure $\varepsilon / \Delta \sim$ $(g / \ln g)^{1 / 2}$ and $\varepsilon / \Delta \sim g^{1 / 2}$ are associated with the decay of single quasi-particle. These thresholds were considered in the Refs. [10 14] and their description was not the aim of this paper. However, the methods introduced in Refs. 12,14 for the investigation of below golden rule decay provides us with the useful tool for the investigation of onset of Chaos in complex many-particle excitations also.

Technically our consideration is based on the two simple equations (5) and (7) for the IPR. The two noninteracting states are strongly mixed by the interaction if the corresponding energy difference turns out to be of the same order of magnitude with the matrix element. As a result, all the effects which we consider are determined by the product of the averaged modulus of the matrix element and the density of accessible states.

The emergency of chaos in the majority of complicated states with many excited particles and holes was the main subject of this paper. This increase of chaos with the growth of excitation energy proceeds in a very complicated way, both technically and even more logically. In the simplest variant, mixing of the simple (single Slater determinant) states is caused by the direct interaction. The mixing by direct interaction opens at $\mathcal{E}>\Delta g^{2 / 3}$ [25]. Due to a large number of excited particles the interaction may proceed through a large number of independent channels. This results in the exponentiation of 
the IPR, as we have shown at the end of the Sec. 2. Thus even the direct interaction may mix together an exponentially large number of Slater-determinant configurations. However, this number is still small compared to the total number of levels of complicated system and the direct interaction could not cause the transition from Poisson to Wigner-Dyson statistics of energy levels.

An alternative to direct interaction is the transition via effective three-particle, four-particle, etc. interaction considered in Sec. 3. The effect of this high-order interaction is much weaker than that of the direct interaction at low energies, but they became comparable at $\mathcal{E} \sim \Delta g / \ln g$. Above the energy $\mathcal{E} \sim \Delta g / \ln g$ our perturbative approach is no more valid and we were forced to introduce the new unknown scaling function $F(\ln g \mathcal{E} / g \Delta)$ into the exponent describing the IPR in the Eq. (17). However, if one expects that $F(x \sim 1) \sim 1$, the logarithm of the number of components in the typical wave function at $\mathcal{E} \sim \Delta g / \ln g$ will be in $\ln g \gg 1$ times smaller than the logarithm of the total number of states. This means that although the mechanism of mixing is changed and the number of components in the wave function became to grow much faster at $\mathcal{E} \sim \Delta g / \ln g$, this threshold does not correspond to the transition Poisson-Wigner-Dyson.

The Wigner-Dyson statistics becomes evident only at $\mathcal{E} \sim \Delta g$. This energy is analogous to the golden rule threshold $\varepsilon \sim \Delta g^{1 / 2}$ for the decay of single quasi-particle. Roughly speaking above the $\mathcal{E} \sim \Delta g$ any particle from the strip $\sim T$ around $\varepsilon_{F}$ may decay via the golden rule.

Formally, in order to understand, how the change of statistics proceeds between $\mathcal{E} \sim \Delta g / \ln g$ and $\mathcal{E} \sim \Delta g$ one should analyze the high order behavior of the coefficients $F_{n}$ in the expansion of the function $F(x)$ Eq. (17). Surprisingly, this analysis in the Sec. 4 instead of such understanding led us to the crucial revision of the considered above straightforward scheme.

First of all, as we have shown in the Sec. 4, the series in the Eq. (17) most likely is the asymptotic series. Although (21) $F_{n} \sim n$ ! is only the upper bound for the $F_{n}$, it seems very unlikely that the factorial divergency of the coefficients may be washed out. Moreover, even if $F_{n} \sim(\nu n)$ ! with any $0<\nu<1$, the results of the Sec. 4 will be only slightly modified. The factorial divergency of the series would not be dangerous if one will sum it up e.g. via some variant of the Borel method. However, within the Borel prescription one is immediately faced with the problem of strange nonperturbative corrections like in the Eq. (22) (moreover that $F_{n}$ are positive by construction and the series is non-Borel summable). Being unable to find the physical motivation for the Borel summation, we were looking for other way to make sense of the divergent series. The coefficient $F_{n}$ is given by the sum of $n+1$-st order tree type Feynman diagrams. Therefore, the natural cutoff for the series is given by the largest diagram which may be drown with available $\sim T / \Delta$ particles. Namely $n_{\max } \sim T / \Delta$ (one more way to determine $n_{\max }$ considered in the Sec. 4 leads to the same physical result). Within this scenario the $n_{\max }$ and the scaling parameter $x=\ln g \mathcal{E} / g \Delta$ in the Eq. (17) are two independent variables. At $x>1 / n_{\max }$ summation in the Eq. (17) goes above the smallest term. However, up to the energy $\mathcal{E}_{c} \sim \Delta(g / \ln g)^{2 / 3}$ all $n_{\max }$ terms of the series are small. At $\mathcal{E}=\mathcal{E}_{c}$ even the broken series blows up and above $\mathcal{E}_{c}$ our perturbative approach is no more valid. Because of the growth of $F(x)$ at $\mathcal{E} \approx \mathcal{E}_{c}$ proceeds mostly due to the last terms of the series or the most complicated diagrams of the highest allowed order of perturbation theory, this threshold corresponds to narrow phase transition from Poisson to Wigner-Dyson spectrum. We have attempted at the end of the previous section to find remnants of non-chaotic behavior above $\mathcal{E}_{c}$ by considering the short time processes. However, this issue requires a separate and more careful investigation.

To summarize, we have considered in this paper the two scenarios, how the quantum chaos may develops in the Finite Fermi systems depending on the way one will use for the summation of factorially divergent contributions from the high order effective interaction.

- If the series is summed up within the Borel prescription (i. e. broken effectively at the smallest term) we were able to find a few distinct stages of the chaotic behavior. Below the energy $\sim \Delta g^{2 / 3}$ the majority of eigenvectors is almost unperturbed. Between $\sim \Delta g^{2 / 3}$ and $\sim \Delta g / \ln g$ direct interaction mixes together the exponentially large number of simple states (7). However, the IPR is under theoretical control in this region and the statistics is clearly Poissonian. Above $\Delta g / \ln g$ statistics of the energy levels is still Poissonian, but the IPR is described by some (unknown) scaling function $F(x)$ (17). Transition to Wigner-Dyson takes place somewhere between $\mathcal{E} \sim \Delta g / \ln g$ and $\mathcal{E} \sim \Delta g$ (see the Eq. (19)). The transition may be relatively narrow $\Delta \mathcal{E} / \mathcal{E} \sim(\ln g)^{-1}$.

- In the second variant the cutoff of the series in the Eq. (17) is determined by the number of the particles in the largest allowed Feynman diagram and is independent of the scaling variable $x$. In this case the transition to Wigner-Dyson takes place at $\mathcal{E}_{c} \sim \Delta(g / \ln g)^{2 / 3}$ and is narrow like $\Delta \mathcal{E} / \mathcal{E} \sim$ $\Delta / T \sim(\ln g / g)^{1 / 3}$.

To our current understanding, the second scenario is more physically motivated. However, our argumentation in this part is not completely rigorous and one still may find the reasons for summation a la Borel of the tree diagrams. The important disadvantage (or advantage?) of the second scheme is that if it is correct, it will be probably the first example of the phase transition caused by the factorial divergency of the series. Finally, although both of our scenarios are reach of new physical predictions, we 
were able to make only the order of magnitude estimates of the considered thresholds. Therefore, any experimental confirmation (within the true experiment, or by numerical simulations) of the mechanisms of development of chaos considered in this paper are very desirable.

Acknowledgments. Author is thankful for discussions to V.F.Dmitriev, V.V.Flambaum, A.A.Gribakina, G.F.Gribakin, I.V.Ponomarev, D.V.Savin, V.V.Sokolov, O.P.Sushkov, V.B.Telitsin, and A.S.Yelkhovsky. The work has been supported in part by the Gordon Godfry foundation and by RFBR, grant 98-02-17905.

[1] E. P. Wigner, Ann. Math. 62, 548 (1955); 65, 203 (1957).

[2] J. B. French and S. S. M. Wong, Phys. Lett. 33B, 447 (1970); Phys. Lett. 35B, 5 (1971).

[3] O. Bohigas and J. Flores, Phys. Lett. 34B, 261 (1971); Phys. Lett. 35B, 383 (1971).

[4] M. Horoi, V. Zelevinsky and B. A. Brown, Phys. Rev. Lett. 74, 5194 (1995); V. Zelevinsky, B. A. Brown, N. Frazier and M. Horoi, Phys. Rep. 276, 85, 1996.

[5] V. V. Flambaum, G. F. Gribakin and F. M. Izrailev, Phys. Rev. E 53, 5729 (1996).

6] D. Weinmann, J.-L. Pichard and Y. Imry, condmat/9705113.

[7] Ph. Jacqoud and D. L. Shepelyansky, cond-mat/9706040; B. Georgeot and D. L. Shepelyansky, cond-mat/9707231.

[8] T. Guhr, A. Müller-Groeling and H. A. Weidenmüller, cond-mat/9707301, Phys. Reports (to appear)

[9] U. Sivan, F. P. Milliken, K. Milkove, S. Rihston, Y. Lee, J. M. Hong, V. Boegli, D. Kern and M. deFranza, Europhys. Lett. 25, 605 (1994).

[10] U. Sivan, Y. Imry and A. G. Aronov, Europhys. Lett. 28, 115 (1994).

[11] Ya. M. Blanter, Phys. Rev. B 54, 12807 (1996).

[12] B. L. Altshuler, Y. Gefen, A. Kamenev and L. S. Levitov, Phys. Rev. Lett. 78, 2803 (1997).

[13] A. D. Mirlin and Y. V. Fyodorov, Phys. Rev. B 56, 13393 (1997).

[14] P. G. Silvestrov, Phys. Rev. Lett. 79, 3994 (1997).

[15] We suppose that the Fermi energy is large compared to the excitation energy $\varepsilon_{F} \gg \mathcal{E}$. The number of particles goes to infinity but experimental accuracy still allows to resolve individual energy levels (the mesoscopic limit).

[16] A. Bohr and B. R. Mottelson, Nuclear Structure, Benjamin, New York 1, 284 (1969).

[17] The temperature and total energy may be easily related via $\mathcal{E}=\int\left(\varepsilon-\varepsilon_{F}\right) \overline{n(\varepsilon)} d \varepsilon / \Delta$.

[18] I. V. Ponomarev, P. G. Silvestrov, Phys. Rev. B 56, 3742 (1997).

[19] V. V. Flambaum, F. M. Izrailev, Phys. Rev. E 56, 5144 (1997).

[20] We use the concept of the distance in the Fock space introduced in Ref. 12. The distance between two simple states equals to one if they may be related by the direct interaction in the first order of perturbation theory.

[21] This combinatorial calculation was done by Cayley more then century ago. Author is thankful to A. Gribakina and G. Gribakin for discussion of this result.

[22] Large order behavior of perturbation theory, edited by L. C. Le Guillou and J. Zinn-Justin (North-Holland, Amsterdam, 1990).

[23] Only if the factorial divergency of $F_{n}$ will be washed out completely due to the large number of coinciding diagrams in Eq. 20) (which seems very unlikely) $\mathcal{E}_{c}$ will be raised again to that predicted by the Eq. (19).

[24] See for example the Appendix B in the lecture by P. A. Mello in:Mesoscopic Quantum Physics Les Houches, Session LXI 1994 (Elsevier, Amsterdam, 1995)

[25] In particular this means that the decay of single quasiparticles starts at parametrically lower energies than the mixing of complicated many-particle excitations $\left(\Delta g^{1 / 2} \ll \Delta g^{2 / 3}\right)$. Thus below the energy $\varepsilon \sim \Delta g^{2 / 3}$ one may consider the decay of quasi-particle (via the golden rule, or not) into almost nonperturbed few particles states as it was done in Refs. [10 14].

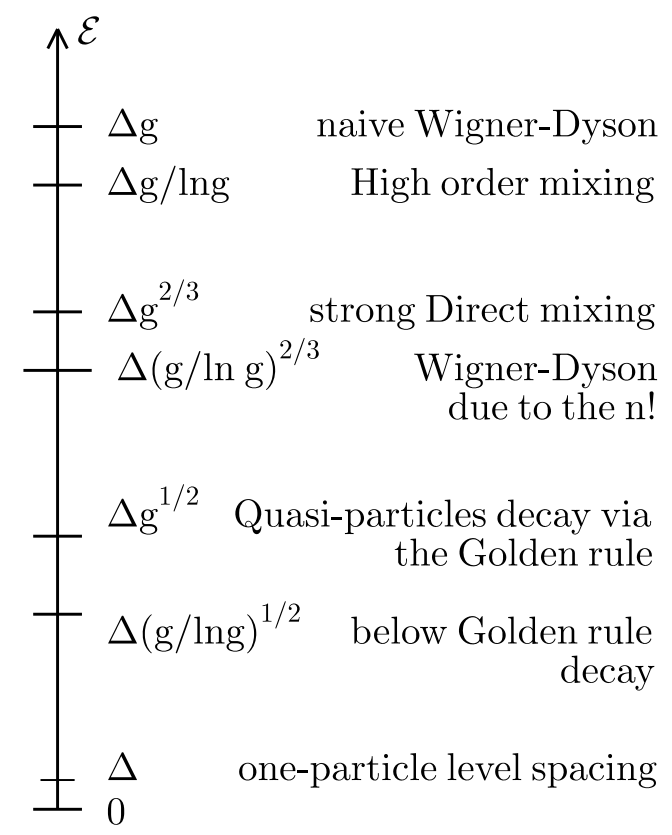

FIG. 1. The different energy scales corresponding to chaotization of single- or many-particle excited states. 


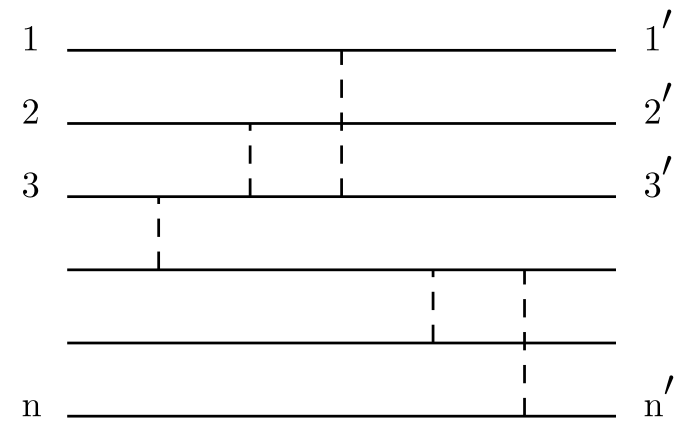

FIG. 2. The tree type diagram contributing to high order effective matrix element. The screened Coulomb interaction is shown by dashed lines. 